UDC 81'27

DOI 10.18485/primling.2015.16.4

\author{
Ksenija Djordjević Léonard \\ EA-739 Dipralang \\ Université Paul-Valéry Montpellier (France)
}

\title{
LANGUES EN DANGER ET REVITALISATION: TYPOLOGIE CONTRASTIVE
}

\begin{abstract}
Résumé: Nous aborderons, dans cette communication, la problématique des langues menacées ou en danger, en Europe et dans le monde. Un aspect souvent oublié de cette question est que les minorités linguistiques, dont les langues ou l'héritage culturel sont menacés, ne restent pas passives face à ce phénomène : elles développent de multiples stratégies de résistance ou de résilience. De ce point de vue, le linguiste joue un rôle important, en tant qu'expert, mais aussi à travers les ressources qu'il produit pour le public (grammaires, dictionnaires, bases de données en ligne, etc.). La synergie entre l'apport du linguiste et les capacités d'organisation et de résilience des communautés débouchent sur cette nébuleuse de pratiques et de solutions techniques qu'est la revitalisation, que nous illustrerons à travers quatre études de cas.
\end{abstract}

Mots-clés: linguistique appliquée, revitalisation, langues en danger, sociolinguistique, langues du monde

\section{Introduction}

La question des langues menacées ou en danger n'est pas seulement une " mode », mais un véritable problème de société, lié au développement de la mondialisation, à la globalisation des échanges, mais aussi aux relations de pouvoir inégal entre communautés d'intérêts. Nous nous interrogerons ici, dans un premier temps, sur les critères qui permettent de juger de la vulnérabilité des langues du monde, avant de présenter les principaux lieux où la diversité linguistique semble condamnée à plus ou moins long terme. Dans un deuxième temps, nous choisirons quelques exemples, à notre sens singulièrement parlants, qui montrent ce que les linguistes font et sont capables de faire sur le terrain, avec la participation de la communauté linguistique. En effet, aux côté des minorités qui ne restent pas passives face aux menaces qui pèsent sur leurs langues et développent de multiples stratégies de résistance ou de résilience, le linguiste joue un rôle important, en tant qu'expert, mais aussi à travers les ressources qu'il produit pour le public. C'est précisément la synergie entre l'apport du linguiste et les capacités d'organisation et de résilience des communautés qui débouche d'ailleurs sur cette nébuleuse de pratiques et de solutions techniques qu'est la revitalisation. 


\section{Vulnérabilité des langues du monde}

\subsection{Attrition linguistique hier et aujourd'hui}

L'intérêt pour les questions liées aux langues en danger et leur possible sauvegarde est d'autant plus grand que le phénomène de l'attrition linguistique, ou disparition des langues, s'accélère à l'époque moderne : près de la moitié des langues du monde sont menacées d'extinction à plus ou moins court terme (Kristal 2003 : 34 ; Hagège 2002 : 9). Cependant, le phénomène n'est pas nouveau et s'est déjà produit au cours de l'histoi$\mathrm{re}^{1}$. Néanmoins, si lors des plus récentes périodes d'extinction massive, comme l'époque coloniale, l'idéologie dominante cautionnait ce processus en le décrétant « facteur de progrès », il n'en est rien aujourd'hui, même si la même rhétorique est parfois encore en œuvre, vantant les bienfaits d'une seule et unique forme de globalisation possible. Les sociétés sont tout de même aujourd'hui bien plus informées et organisées pour résister aux différentes formes d'agression par des intérêts géo-financiers et politiques que ne l'étaient, lors des «grandes découvertes européennes », les peuples des pays tropicaux qui allaient subir le joug colonial après une conquête violente, légitimée par une idéologie verticale, que seules les composantes les plus conservatrices de nos sociétés modernes continuent encore de cautionner.

En ce qui concerne l'époque moderne, on pourrait avoir l'impression que la réflexion sur la diversité linguistique s'est développée avec la publication de livres tels que Halte à la mort des langues de Claude Hagège, Language Death de David Crystal ou Vanishing Voices. The Extinction of the World's Languages de Daniel Nettle et Suzanne Romaine. Ces trois essais ont contribué, chacun à sa façon, à une prise de conscience auprès du grand public ou de ce qu'il est convenu d'appeler « le public éclairé », de la nécessité de lutter contre la disparition des langues. Il ne faut pas pour autant oublier que dès les années 1970, Joshua A. Fishman, dans ses travaux sur la diglossie, apportait une pierre de touche à cette réflexion, qu'il développera plus tard avec le concept de $R e$ versing Language Shift (1991). Il s'agit d'un processus de retournement de substitution linguistique, qui consiste pour une langue à se maintenir en vie, et à réapparaitre sur le devant de la scène, alors que tout la destinait à disparaître - un phénomène de réversibilité active et volontaire. En réalité, un certain nombre d'articles importants paraissaient sur cette question depuis le début des années 1990, notamment dans la revue nord-américaine Language. On y trouve, par exemple, des textes de quelques-uns des plus grands linguistes contemporains, comme Ken Hale, Akira Y. Yamamoto, Nora C. England, Nancy Dorian, et d'autres².

On remarquera que les termes utilisés à l'époque moderne pour parler de l'attrition

1 Il suffit de penser aux langues anciennes de la famille indo-européenne : le hittite d'Anatolie (disparu au XII ${ }^{\text {ème }}$ siècle av. J-C.), le tokharien du Turkestan chinois (disparu au IX ${ }^{\text {ème }}$ siècle de notre ère), et encore le latin ou le grec ancien.

2 Cf. notamment le ${ }^{\circ} 68$ de 1992 . Certains de ces linguistes avaient pu observer d'ailleurs de très près le processus d'agonie des langues (comme Nancy Dorian face à l'attrition du gaélique d'Ecosse dans une île très périphérique des Highlands), ou avaient été témoins plus ou moins directement de l'oppression dont des populations autochtones d'Amérique centrale avaient fait l'objet sous la coupe de dictateurs (comme Nora C. England, spécialiste du mam et des langues mayas du Guatemala, ou Colette Craig - Grinevald -, spécialiste de jakaltek, ou popti', selon l'ethnonyme endogène). 
linguistique, y compris dans les titres des ouvrages cités ci-dessus, sont le plus souvent la « vie » et la «mort » des langues. L'usage de ces métaphores peut être vu comme l'héritage du XIX ${ }^{\text {ème }}$ siècle, marqué par les idées de Darwin, où les comparaisons entre les langues et les espèces vivantes étaient fréquentes. Si la métaphore a le mérite d'être explicite, il existe en effet toute une série de termes qui correspondent à des situations et des cas de figure bien précis : attrition (diminution de l'espace social d'une langue), obsolescence (caractère désuet projeté sur la langue et évitement fonctionnel dans la communication courante), extinction (disparition ou effacement de la langue), substitution (remplacement d'une langue minoritaire par une langue majoritaire, le plus souvent officielle), etc.

\subsection{Critères de vitalité et attitude des locuteurs}

La question de la disparition des langues et de leur possible revitalisation semble préoccuper de nos jours également les institutions internationales. Le rôle de l'Unesco apparaît comme central dans ce domaine. Cette organisation des Nations Unies a inauguré, autour de l'an 2000, des actions de protection des langues menacées, et a entrepris une étroite collaboration avec certains linguistes connus pour avoir réfléchi à ces questions, dont, par exemple, Matthias Brenzinger, Colette Grinevald, Michael Krauss, Arienne Dwyer, etc. Sur le site de l'Unesco consacré au programme sur les langues en danger, on trouve un «Atlas des langues en danger dans le monde $»^{3}$, avec des cartes interactives, d'une grande utilité scientifique et pédagogique. L'Atlas propose diverses entrées possibles : par pays ou région, par nom de la langue, son code international, selon le nombre de locuteurs et le degré de vitalité ou de danger de disparition.

Les linguistes qui ont collaboré à ce projet ont élaboré une "typologie » de la vitalité des langues du monde. Les six niveaux de vitalité d'une langue - sûre, vulnérable, en danger, sérieusement en danger, en situation critique et éteinte - vont de celui considéré comme le plus positif (langue en « bonne santé », dont la vitalité est assurée et l'avenir non compromis) au critère le plus préoccupant (langue déjà disparue). Le niveau de vitalité d'une langue est déterminé en fonction des critères suivants qui sont autant de facteurs cruciaux pour l'avenir d'un idiome : taux de locuteurs sur l'ensemble de la population, nombre absolu de locuteurs, transmission de la langue d'une génération à l'autre, attitude des membres de la communauté vis-à-vis de leur propre langue, utilisation de la langue dans les différents domaines publics et privés, attitudes et politiques linguistiques au niveau du gouvernement et des institutions, usage et statuts officiels, type et qualité de la documentation, réaction face aux nouveaux domaines et médias, disponibilité de matériels d'apprentissage et d'enseignement des langues. Les neuf critères retenus par les experts de l'Unesco entretiennent une relation de dépendance et de lien mutuel.

Parmi les neuf critères de l'Unesco, le plus important pour la question qui nous préoccupe ici est celui de l'attitude des locuteurs vis-à-vis de leur langue. Certes, la communauté linguistique ne peut pas être considérée comme un ensemble uni à tous points de vue. Quand il s'agit de la volonté de revitaliser un idiome vulnérable ou en voie de disparition, au sein d'une même communauté on peut observer des attitudes différentes : motivation, disponibilité à travailler avec les linguistes, rejet des initiatives venues de

3 http://www.unesco.org/languages-atlas/fr/atlasmap.html. 
l'extérieur, rejet des initiatives locales, etc. L'attitude des locuteurs est à mettre également en relation avec les conditions de vie. En effet, on comprend aisément que la langue ne fait pas toujours partie des priorités d'un groupe minoritaire, minoré, dévalorisé, déconsidéré. Si la communauté est motivée, le linguiste peut avoir un rôle à jouer, dans la synergie entre les instances de décision et les locuteurs. Cependant, la revitalisation, telle que nous l'entendons, est tout le contraire d'une démarche descendante, voire condescendante : il ne s'agit pas d'imposer des normes culturelles ni l'usage étendu de la langue à tous prix, mais de proposer des solutions à des problèmes de minoration socioculturelle, politique et idéologique, en favorisant une réactivation des vecteurs d'interaction et de solidarité au sein de sociétés qui subissent une forte pression de l'extérieur sur leurs ressources naturelles et humaines. Il s'agit donc d'une action sociale et d'un travail social.

\section{Lieux et causes de l'attrition linguistique}

De nombreuses cartes permettent aujourd'hui d'identifier les territoires les plus vulnérables sur le plan de la diversité linguistique. Ainsi, sur la carte consultable sur le site de l'Unesco ${ }^{4}$ on repère dans les grandes lignes les régions dotées de la plus grande densité linguistique. On n'est guère étonné de constater que c'est dans ces mêmes régions que l'on trouve également le plus grand nombre de langues indiquées avec des couleurs foncées - donc vulnérables ou en danger ${ }^{5}$. Les régions d'une grande densité et d'une grande vulnérabilité sont surtout, d'après la carte, la ceinture tropicale de l'Afrique, le nord de l'Australie, la façade pacifique de l'Amérique du sud et de l'Amérique centrale et les îles de l'océan Pacifique. Pour les territoires qui nous sont proches (Europe), la densité linguistique est beaucoup plus faible, les langues sont pour la plupart « en bonne santé », mais ce n'est pas pour autant que notre continent ne compte pas un certain nombre de langues en voie de disparition, ou même déjà disparues ${ }^{6}$. En France, de nombreuses langues régionales, notamment les langues d'oïl (bourguignon-morvandiau, lorrain, champenois, gallo...), sont aujourd'hui en grande difficulté, ou pour reprendre la nomenclature de l'Unesco, elles sont déjà « sérieusement en danger ».

Comment les langues se retrouvent dans une situation d'attrition irréversible, sur le plan de leur sociabilité ? Deux auteurs cités précédemment, C. Hagège et D. Crystal, dans leurs ouvrages respectifs, proposent quelques éléments de réponse. C. Hagège évoque trois causes possibles : les causes physiques, les causes économiques et sociales, et les causes politiques (2002:119-191). David Crystal, pour sa part, distingue entre deux types de facteurs : les facteurs physiques et les facteurs culturels (2003 : 97-126). Dans le premier cas - facteurs physiques -, une langue meurt avec ses locuteurs, suite aux catastrophes naturelles, tsunamis, tremblements de terre, épidémies, éruptions volcaniques, ouragans, etc. Les deux auteurs donnent beaucoup d'exemples pour illustrer ces cas de figure. Ainsi, par exemple, le tremblement de terre en Papouasie-Nouvelle-Guinée, en

4 http://www.unesco.org/languages-atlas/fr/atlasmap.html

5 En effet, les auteurs de l'Atlas ont choisi de représenter la vitalité linguistique avec une couleur différente pour chaque niveau : plus les couleurs sont foncées, plus la langue est en danger.

6 Par exemple, le slovince en Pologne, le dalmate en Croatie, le carélien de Valday en Russie, le karaïm en Ukraine, etc. 
1998, a-t-il modifié le paysage linguistique de ce territoire, dans la mesure où plusieurs villages dont les langues étaient en cours d'étude ont été rasés de la surface de la terre (Kristal 2003 : 101). Parmi les raisons physiques, on peut citer également la famine, provoquée par exemple par la sécheresse (ex. Sahel 1983-1985, Somalie 1991-1992) (Kristal 2003 : 102). Des proportions considérables de populations autochtones d'Amérique ont succombé aux maladies diffusées par les colons, même celles désormais faciles à soigner, comme la grippe. Plus récemment, d'autres fléaux sociaux, comme l'alcoolisme et la dépendance à la cocaïne font des ravages dans nombre de communautés linguistiques d'Amazonie de faible masse démographique. Enfin, l'exploitation des ressources naturelles que recélaient les territoires de grands réservoirs de diversité linguistique a également parfois mené jusqu'aux conflits territoriaux dans lesquels les populations locales ont péri. C'est ce qui est arrivé aux Yanomami du Brésil à la fin des années 1980 lorsque leur territoire a été envahi par les milliers des chercheurs d'or, semant la panique, exerçant une violence constante, dégradant de façon durable l'habitat et l'environnement des Indiens, les privant de leurs moyens de subsistance et transmettant des maladies mortelles pour ces populations (rougeole, grippe, tuberculose, paludisme...), comme le décrivent Davi Kopenava et Bruce Albert dans l'essai anthropologique La chute du ciel, publié en 2010. Le deuxième type de facteurs analysés par David Crystal (2003 : 108-109) - facteurs culturels - illustre une situation différente : une langue meurt, mais les locuteurs continuent à vivre. Il s'agit dans ce cas-là d'une assimilation, soit par le groupe démographiquement le plus important, soit par le groupe économiquement dominant. Ce processus se lit également comme une acculturation au profit de la majorité de plus en plus dominante, au fil des générations, menant à l'atrophie et la résorption des langues en situation minoritaire.

\section{Linguistique de terrain : études de cas}

De nos jours, de nombreux linguistes et sociolinguistes s'orientent vers l'observation des situations sociolinguistiques dans lesquelles on peut identifier les langues en danger de disparition et la description de celles-ci. Leur travail ne se limite pas à faire le constat, mais implique nécessairement une action. Celle-ci peut prendre plusieurs formes. Il convient en effet de distinguer entre quatre axes principaux de l'action des linguistes : description, documentation, archivage et revitalisation (Bert et Grinevald 2010 : 118). Les trois premières tâches - description, documentation, archivage - concernent le corpus. Il s'agit de recueillir la parole et de collecter des données sur le terrain, afin de les conserver pour l'avenir. Le dernier axe - la revitalisation - consiste à empêcher la substitution sociolinguistique.

Selon J.L. Léonard (2009 : 57-58), il y a au moins trois bonnes raisons de défendre les langues minoritaires, et cela est valable également pour les langues en situation de minoration extrême qui nous intéressent ici : le libre arbitre, la contre-histoire et le pluralisme socioculturel. Le libre arbitre implique que chacun reste libre de ses choix, y compris celui de parler sa langue maternelle, tant que ce choix n'empiète pas sur la liberté d'autrui. La règle qui prédomine depuis des siècles, et qui ne fait qu'empirer avec la posture postmoderniste, est précisément le principe inverse : on considère comme naturel d'empiéter sur la liberté des communautés linguistiques minorisées de continuer à utiliser 
leurs langues, ou encore d'élaborer leur corpus ou de valoriser leurs fonctions, dans le cadre d'une autonomie culturelle qui revêt souvent une fonction d'autoprotection face à la prédation globalisée - quand elles parviennent tant bien que mal à aménager une telle marge d'action. La contre-histoire est illustrée par la résistance d'une langue à la substitution par une autre langue en tant que résistance à un ordre inégalitaire, comme la situation coloniale - ou postcoloniale. Bien souvent, en remontant l'écheveau historique d'une situation d'acculturation locale, on retrouve toute la trame d'une politique erronée ou injuste, ou d'une forfaiture. Enfin, les langues nous fournissent des ressources différentes, et permettent ainsi l'expression d'un point de vue différent en termes de complémentarité socioculturelle, mais aussi en termes de projets de société. Favoriser le pluralisme socioculturel dans le cadre d'une démocratie participative devrait rester un idéal de gouvernance. Ces trois raisons sont autant de conditions nécessaires à la coexistence pacifique et à l'équilibre démocratique entre majorité et minorité.

Les différents exemples qui suivent montrent l'extension géographique des entreprises de revitalisation, phénomène de plus en plus présent dans notre monde globalisé. Nous avons essayé de diversifier autant que possible les langues et les familles linguistiques représentées ${ }^{7}$.

\subsection{Le vepse (Russie)}

Le vepse est une langue finno-ougrienne minoritaire parlée au nord-ouest de la Russie, notamment en République de Carélie et dans les oblasts de Leningrad et de Vologda. Les 5936 Vepses de Russie - dont 3613 se déclarent encore comme locuteurs de la langue ${ }^{8}$ - ne bénéficient pas d'une véritable reconnaissance juridique et linguistique. En effet, le vepse n'a pas de statut de langue officielle, ou co-officielle avec le russe, ni en Carélie, ni ailleurs sur le territoire russe où habite cette minorité. Cependant, en République de Carélie, le vepse est protégé et valorisé en tant qu'un élément de patrimoine, au même titre que le carélien ou encore le finnois. La visibilité et la présence de la langue vepse sont certes minimes, tant cette langue se trouve objectivement dans une dynamique sociolinguistique défavorable. Nous avons écrit par ailleurs (Djordjevic Léonard 2013 : 87-99), suite à un séjour de terrain en avril-mai 2013 au sein de la population vepse septentrionale ${ }^{9}$, que la situation sociolinguistique de cette langue laissait à désirer : elle est absente de l'administration, son usage dans le domaine public est pratiquement inexistant - excepté quelques concessions symboliques comme l'affichage bilingue dans les villages vepses, son enseignement reste rare et facultatif, tandis que le domaine médiatique et éditorial peine à lui trouver une place.

Pourtant, cette langue a su profiter d'un certain réveil de la conscience nationale qui a marqué les populations finno-ougriennes dans les années 1990. Les différentes mesures prises à cette époque en vue de la protection de la langue vepse en Russie ont permis certaines améliorations dans l'aménagement linguistique et initié diverses me-

7 Le lecteur trouvera d'autres exemples dans Costa, 2013, Léonard \& Avilés Janiré González, 2015, ou encore, une fois n'est pas coutume, dans un livre journalistique : Abley, 2006.

8 Ces chiffres correspondent au dernier recensement russe de 2010. Cf. http://www.gks.ru/.

9 La zone septentrionale du peuplement vepse correspond à une étroite région lacustre, près du lac Onega en Carélie, à proximité de la ville de Petrozavodsk. 
sures en vue de sa revitalisation, notamment dans la zone vepse septentrionale, et dans la capitale carélienne, Petrozavodsk. La langue est rentrée dans le système universitaire, avec la création de la Chaire de la langue et de la culture vepses et caréliennes ; les « nids de langue » ont donné des résultats encourageants au niveau de l'école maternelle ne serait-ce qu'à travers l'image positive redonnée de la langue maternelle ; la recherche universitaire s'est développée, notamment en histoire, ethnologie, linguistique ; le journal bilingue Kodima, malgré son tirage modeste, joue encore aujourd'hui un rôle symbolique non négligeable. Tout cela a été possible grâce au travail et à l'action des aménageurs activistes et militants de la cause vepse - essentiellement de Petrozavodsk. Réunis autour de l'Institut de langue, de littérature et d'histoire de Carélie, les chercheurs locaux mènent depuis plus de deux décennies maintenant un véritable combat pour la sauvegarde de la langue vepse, comme nous l'avons montré dans un travail récent sur la mise en visibilité de la langue vepse (2014 : 221-232). Dans ce texte, basé sur nos observations de terrain, nous expliquons comment le travail de revitalisation s'effectue au sein d'un dense réseau d'activistes dont les membres font constamment le lien entre l'enseignement, les médias et la recherche d'une part, et les usagers de la langue d'autre part. A cela s'ajoutent des professionnels du domaine médiatique, qu'ils travaillent dans la presse (journal Kodima), ou le domaine audio-visuel (radio, télévision), ou encore dans celui des nouvelles technologies, que ce soit à travers l'élaboration des outils didactiques ou le travail de documentation. On observe une forte interaction et rétro-alimentation aussi bien technique que pratique entre aménagement linguistique « de par en haut» (démarche descendante), « de par le bas » (démarche ascendante) et à « mi-palier ».

Le travail de ce petit groupe de personnes engagées dans la revitalisation du vepse a de quoi susciter l'admiration, tant elles ont réussi à créer une véritable synergie entre le milieu des (derniers) locuteurs et celui des chercheurs et des pédagogues. La situation sociolinguistique est, certes, celle d'une langue désormais bien engagée vers la substitution par le russe, tout comme les Vepses sont de plus en plus assimilés par la population majoritaire, mais ces aménageurs locaux ont toutefois réussi à créer un véritable monde de connaissances, de productions et de réalisations de tous points de vue originales, qui assure encore un certain avenir à la langue vepse. Du moins à court terme.

\subsection{L’hébreu (Israël)}

Le cas de l'hébreu représente certainement l'exemple le plus connu et le plus remarquable de ce que l'on pourrait appeler une résurrection linguistique. Cependant, c'est aussi, par la dimension du phénomène et son histoire événementielle et politique, une singularité - c'est-à-dire un phénomène puissant, mais si particulier et difficile à reproduire en d'autres circonstances qu'on ne saurait en faire un parangon. Il a fallu avant tout la volonté inébranlable d'un homme pour que cette langue - dont l'usage était uniquement écrit et religieux - s'impose de nouveau comme une langue parlée. Cet homme, Eliézer Ben Yéhouda, est considéré comme le père de l'hébreu moderne. Lexicographe, né dans la diaspora, en Lituanie, il a consacré sa vie à cette langue « des textes » qu'il voulait faire revivre. La réalisation de cette tâche était rendue possible notamment parce que les témoignages écrits étaient nombreux, et la conscience historique de la communauté, bien développée. Quelques pionniers l'ont suivi dans son enthousiasme, et ont réussi à 
insuffler peu à peu de la « vie » à cette langue « morte». Des écoles hébraïques ont été mises en place à cette fin, et elles ont contribué, à leur tour, à donner aux Juifs une identité collective, d'abord symbolique, avant de devenir bien réelle et de se généraliser dans son domaine culturel.

En effet, un projet culturel repris par le sionisme ${ }^{10}$ fera de la question linguistique une question identitaire, la langue devant permettre la différenciation du groupe par rapport aux autres. Il a fallu beaucoup de rigueur, un volontarisme infatigable, et une discipline de tous les instants : Ben Yéhouda va aller jusqu'à obliger sa femme à parler à leurs enfants uniquement en hébreu, aussi imparfait ou inadapté au monde moderne que fût encore cette langue à l'époque, dans l'état de réélaboration et de refonctionnalisation où elle se trouvait encore. Son comportement est jugé par certains comme relevant d'un « volontarisme fanatique » (Dieckhoff 2002 : 274). On peut imaginer facilement à quel point la nécessité d'enrichir le vocabulaire était grande, après deux millénaires de confinement de la langue dans les livres, à quel point la syntaxe était encore inadaptée aux besoins de la langue du quotidien. Il est également auteur du dictionnaire - qu'il n'a pas pu achever de son vivant - de la langue moderne, avec les mots nécessaires pour son usage quotidien.

C. Hagège écrit au sujet de l'hébreu moderne : "Ainsi, les langues mortes sont des structures sans voix, mais non sans existence. Il "suffit" d'un très puissant vouloir collectif et d'une conscience nationale alimentée par un très vif sentiment de continuité bravant les fureurs dissolvantes du temps, pour qu'une résurrection soit possible » (Hagège 2009 : 696). Il convient tout de même de garder à l'esprit l'idée que le cas de l'hébreu est unique, et que même si les minorités linguistiques de par le monde se sont parfois inspirées de ce modèle, personne ne l'a vraiment suivi. On retrouve là la notion de singularité de cette situation, dans le panorama des expériences de revitalisation au cours de l'histoire moderne. La revitalisation des langues de nos jours pourrait difficilement se baser sur des comportements aussi volontaristes, comme celui de Ben Yéhouda, ce qui ne retire rien au mérite de son travail de linguiste, passionné par sa langue et par l'histoire de son peuple.

\subsection{Le maori (Nouvelle-Zélande)}

Le maori, langue austronésienne proche du tahitien et de l'hawaïen, fait partie des langues que l'on peut considérer comme partiellement « sauvées ». La revitalisation a été entreprise avant tout au sein des centres d'éducation de la petite enfance - au sein des « nids de langues ». Elle a commencé dans les années 1980 : cours de langue, écoles intégrant l'enseignement en maori, programme radio. Tout cela s'est fait dans un contexte d'ouverture vers les cultures locales. Mais ces centres ont rencontré des difficultés pour recruter du personnel qualifié, notamment sur le plan linguistique. La langue est certes devenue officielle en 1987, mais l'égalité avec l'anglais reste surtout symbolique. Aujourd'hui, les écoles qui proposent l'enseignement en immersion existent ; une chaîne TV a été créée en 2003, les sites Internet avec l'enseignement de la langue ont un certain succès (Rodd 2014 : 360-361).

Si la politique linguistique dans le domaine des médias est plutôt bien organisée,

10 Idéologie fondée sur le sentiment national juif qui s'est développée à la fin du XIX ${ }^{\text {ème }}$ siècle. 
la société environnante, en dehors de la communauté elle-même, ne se montre guère enthousiaste pour la protection et la sauvegarde de la langue. Cependant, ces différentes initiatives ont tout de même sensiblement amélioré la situation, comme le décrit A. Rodd : le pourcentage de Maoris s'estimant capables de parler « bien ou très bien » le maori est passé de $9 \%$ en 2001 à $14 \%$ en 2006. Même si ce sont surtout les personnes relativement âgées qui le parlent très bien, les chiffres suggèrent un degré de revitalisation chez les plus jeunes : les Maoris âgés de moins de 25 ans en 2001 étaient $6 \%$ à parler très bien la langue, soit deux fois plus que leurs compatriotes de la tranche d'âge supérieure (Rodd 2014 : 365). Le fait que les locuteurs de la langue soient aujourd'hui relativement jeunes a quelque chose d'encourageant. Cela veut dire que ces jeunes assument leurs origines et leur identité culturelle et qu'ils cherchent une valorisation et une reconnaissance venue de l'extérieur. Sur le plan administratif, la présence du maori reste en partie symbolique : la langue est visible dans l'affichage public, mais la présence des Maoris est d'ailleurs plus importante sur l'île située au nord, notamment à Gisborne, tandis que sur l'île située au sud, son usage demeure un fait exceptionnel.

On voit, à travers l'exemple maori, que des efforts soutenus de la part des membres de la communauté maorie, avec l'aide du gouvernement, ont porté leurs fruits sur le plan du retournement de la substitution, pour utiliser le terme de J. Fishman : la langue, même si toujours menacée, est plus vivante et davantage utilisée qu'avant. Les acteurs de sa revitalisation ont su profiter de tous les outils que l'époque contemporaine nous offre, y compris Internet, pour intégrer la langue à la société moderne. Par ailleurs, les techniques de revitalisation utilisées pour favoriser la résurgence de cette langue ont servi et servent encore de modèles ailleurs sur la planète. Le «modèle » s'est exporté, en quelque sorte, avec succès - notamment le concept de "nids de langue ", qui a connu un très grand succès ailleurs dans le monde. Cette diffusion ne s'est pas faite simplement par réputation, mais par une activité systématique de promotion. On voit là un exemple frappant d'activisme non pas seulement centré sur une situation locale, mais également soucieux de partager les fruits d'une méthodologie avec d'autres populations se trouvant dans une situation analogue.

\subsection{Le mohawk (Canada)}

Le mohawk est une langue amérindienne, de la famille iroquoise, dont le territoire se situe entre le Canada (Ontario et Québec) et le nord-est des Etats-Unis (Caroline du Nord). Cette langue est parlée encore par environ 500 personnes au Canada ${ }^{11}$. Dans les années 1970, l'assimilation des Mohawks à commencé à s'accélérer. La prise de conscience de la nécessité de juguler l'érosion linguistique s'opère au début des années 1970, lors de la création des écoles d'immersion au Québec, dans la réserve de Kahnawake, afin d'augmenter la présence de la langue dans la société. La ville abrite également le Kahnawake Language Center. Le territoire s'est doté de sa propre loi linguistique en 2002 , dont le but est de préserver la langue. Plus symbolique que réaliste, cette loi montre l'attachement de la communauté à l'un des éléments de son identité.

Les efforts accomplis en vue de la revitalisation se sont réalisés sur la base du

11 Cf. https://www.ethnologue.com/language/moh. 
volontariat de personnes qui, le plus souvent, ne sont pas des locuteurs natifs, le nombre de ces derniers n'ayant cessé de décroître depuis un siècle. Mais grâce aux programmes d'immersion, aux « nids de langues », aux cours pour les adultes, appuyés sur un fort sentiment identitaire, les différentes entreprises de revitalisation ne rencontrent pas beaucoup de difficulté pour recruter des volontaires. Ce travail a aussi un revers de la médaille : il est périodiquement signalé dans les médias, depuis quelques années, que certains territoires mohawk ne sont pas très ouverts vis-à-vis des étrangers : l'expulsion de résidents non-mohawks, la non-reconnaissance des mariages mixtes, l'interdiction de séjour dans la réserve sont devenues des normes communautaristes récurrentes ${ }^{12}$. Toutes ces décisions aggravent les relations entre les Mohawks et les Québécois, déjà tendues depuis les troubles provoqués par la crise d'Oka dans les années 1990 - dans laquelle on ne peut que donner raison aux Mohawks, puisqu'il s'agissait de raser une partie de leur cimetière afin d'élargir un terrain de golf....

En revanche, le repli identitaire croissant vis-à-vis des non Mohawks résidant dans la communauté s'avère quelque peu embarrassant non seulement pour le gouvernement mais aussi pour les gens impliqués dans la défense et la promotion de la langue - certaines personnes expulsées ces dernières années étaient connues pour s'être beaucoup impliquées en faveur de la cause mohawk elle-même. Cependant, on trouve des tendances analogues dans tous les mouvements à forte identité culturelle qui doivent faire des choix stratégiques face à une politique de répression ou à une guerre de basse intensité avec leur gouvernement.

\section{Conclusion}

Après ce voyage à travers le monde, nous revenons à la question principale : pourquoi chercher à ralentir le processus de l'attrition linguistique, pourquoi chercher à « sauver» les langues? Nous avons donné plusieurs réponses à cette question tout au long de ce texte, si bien que nous pourrions ici n'en ajouter qu'une seule : parce que la diversité, aussi bien biologique que linguistique ou culturelle, est dans la nature même de notre monde, et que préserver cette diversité, c'est aménager l'avenir. Avec chaque langue qui meurt, meurt également une partie de l'humanité et des connaissances uniques sur notre passé, sur les contacts que les populations ont entretenus les unes avec les autres, certes. Mais c'est aussi tout un secteur en lutte pour plus de justice, de vérité historique, d'équilibre écologique, et de raison dans l'histoire qui disparaît. C'est la dimension de la contre-histoire, de la résistance aux hégémonies et à la discrimination, à l'injustice, à la répression et à l'autoritarisme, qui se trouve affaiblie. Le linguiste ne peut pas se permettre d'être un simple observateur : il se doit d'agir. Dans son livre journalistique sur les langues menacées, Mark Abley (2006 : 157) annonce trois phases par lesquelles passe la revitalisation, dont il a été ici souvent question : la sécurité au présent, la confiance dans l'avenir et la curiosité pour le passé. En outre, on pourrait ajouter : la lutte pour un monde meilleur et plus juste.

12 Cf. par exemple http://www.lefigaro.fr/international/2010/02/13/01003-20100213ARTFIG00186-les-indiens-mohawks-ne-veulent-plus-des-blancs-php, et http://ici.radio-canada.ca/nouvelles/societe/2015/05/18/002-couple-mixte-chasse-reserve-kahnawake.shtml. 


\section{Bibliographie}

Abley Marc. (2006). Parlez-vous boro? Voyage au pays des langues menacées. Montréal : Editions du Boréal.

Bert Michel \& Grinevald Colette. (2010). « Proposition de typologie des locuteurs de LED », in Faits de langue, n³5-36, Linguistique de terrain sur langues en danger : Locuteurs et linguistes (coord. M. Bert et C. Grinevald), 117-132.

Costa James (dir.). (2013). Enjeux sociaux des mouvements de revitalisation linguistique, in Langage \& Société, n¹45. Paris : Éditions de la Maison des Sciences de l'Homme.

DIECKHOFF Alain. (2002). «L'invention de l'hébreu, langue du quotidien national », in Denis Lacorne et Tony Judt, La politique de Babel. Du monolinguisme d'Etat au plurilinguisme des pauples. Paris : Karthala, 261-276.

DJORDJEVIC LÉONARD Ksenija. (2013). « Dynamique sociolinguistique de la langue vepse », in Harri Veivo \& Julia Nyikos (dir.), « Marges, interstices, contacts », Cahiers d'Etudes Hongroises et Finlandaises, $\mathrm{n}^{\circ} 19$. Paris : L’Harmattan, 87-99.

« Endangered Languages », Language, $\mathrm{n}^{\circ} 68$.

DjoRDJEVIC LÉONARD Ksenija (dir.). (2014). « La mise en visibilité de la langue vepse en République de Carélie », in Ksenija Djordjevic Léonard (dir.), Les minorités invisibles : diversité et complexité (ethno)sociolinguistiques. Paris : Michel Houdiard Editeur, 221-232.

Fishmann Joshua A. (1991). Reversing Language Shift, Clevedon : Multilingual Matters.

Hagège Claude. (2002 [1 ère éd. 2000]). Halte à la mort des langues. Paris : Odile Jacob.

Hagège Claude. (2009). Dictionnaire amoureux des langues. Paris : Plon.

Kopenava Davi \& Albert Bruce. (2010). La chute du ciel. Paroles d'un chaman yanomami. Paris : Terre Humaine/Plon.

Kristal Dejvid. (2003). Smrt jezika. Beograd : XX vek.

LÉONARD Jean Léo. (2009). " Logique d'élucidation, refondation épistémologique et empirisme critique : pour une linguistique impliquée », in Isabelle Pierozak \& Jean-Michel Eloy (dir.), Intervenir : appliquer, s'impliquer ? Paris : L'Harmattan, coll. « Espaces discursifs », 57-59.

Léonard Jean Léo \& Avilés Janiré González Karla (dir.). (2015), Documentation et revitalisation des «langues en danger » : épistémologie et praxis. Paris : Michel Houdiard Editeur.

Nettle Daniel \& Romaine Suzanne. (2003 [1 1 ére éd. 2000]). Ces langues, ces voix qui s'effacent. Paris : Autrement.

Rodd Adrien. (2014). « Invisibles en Australie, visible en Nouvelle-Zélande ? Enjeux comparés des langues minoritaires autochtones ", in Ksenija Djordjevic Léonard (dir.), Les minorités invisibles : diversité et complexité (ethno)sociolinguistiques. Paris : Michel Houdiard Editeur, 358-370. 


\section{Ksenija Djordjević Léonard}

\section{JEZICI U OPASNOSTI I REVITALIZACIJA: KONTRASTIVNA TIPOLOGIJA}

Sažetak: Ovaj rad se bavi problematikom ugroženih jezika ili jezika u opasnosti, u Evropi i u svetu. U pristupu ovom pitanju, često se zaboravlja da jezičke manjine, čiji su jezici i kulturno nasleđe ugroženi, nisu samo pasivni posmatrači tog procesa : one razvijaju raznovrsne strategije otpora ili rezilijencije. U tom pogledu, lingvista ima važnu ulogu, kao ekspert, ali i zahvaljujući svim resursima koje proizvodi za javnost (gramatike, rečnici, elektronske baze podataka, i sl.). Sinergija između doprinosa lingviste i organizacionih i rezilijentnih sposobnosti zajednica može imati za rezultat revitalizaciju jezika, kao skup praktičnih i tehničkih rešenja. Četiri primera će nam poslužiti za ilustraciju ovog fenomena.

Ključne reči: primenjena lingvistika, revitalizacija, jezici u opasnosti, sociolingvistika, jezici sveta 